\title{
Consensus Statement: Expedition Inspiration Fund for Breast Cancer Research Meeting 2002
}

\author{
Christopher Benz ${ }^{1}$, Gary Clark ${ }^{2}$, Suzanne Conzen ${ }^{3}$, Ronald Dorn ${ }^{4}$, Suzanne Fuqua ${ }^{2}$, Julie \\ Gralow $^{5}$, Geoffrey Greene ${ }^{6}$, Ruth Heimann ${ }^{3}$, Samuel Hellman ${ }^{3}$, Marc Lippman ${ }^{7}$, Neal Rosen ${ }^{8}$, \\ and Louis Weiner ${ }^{9}$ \\ ${ }^{1}$ Buck Institute, Novato, CA $;{ }^{2}$ Baylor College of Medicine, Houston, TX; ${ }^{3}$ University of Chicago Medical Center, \\ Chicago, IL; ${ }^{4}$ Mountain States Tumor Institute, St. Boise, ID; ${ }^{5}$ University of Washington, Seattle, WA; ${ }^{6}$ University \\ of Chicago, Chicago, IL; ${ }^{7}$ University of Michigan Health System, Ann Arbor, MI; ${ }^{8}$ Memorial Sloan-Kettering \\ Cancer Center, New York, NY; ${ }^{9}$ Fox Chase Cancer Center, Philadelphia, PA, USA
}

Key words: breast, cancer, hormones, molecular markers, receptors

\section{Introduction}

The sixth Expedition Inspiration conference was held from February 28 to March 3, 2002 at The Sun Valley Lodge. While there are other conferences that concentrate on a particular facet of breast cancer, the design and goals of this conference are unusual. In order to maximize interaction of investigators and clinicians the meetings are small, invited, and private. The participants include both senior and junior physicians and scientists involved in clinical and basic research as well as clinical practice. Some participants have been at previous Expedition Inspiration meetings while others are new. The meetings serve four purposes:

1. Active discussion among participants who do not usually interact.

2. Develop consensus as to the state of our knowledge on particular aspects of breast cancer to simulate future studies.

3. Develop collaborative projects among the meeting participants.

4. Foster new investigations by participants as well as others.

This year's conference emphasized the real and potential clinical applications of some of the molecular aspects of breast cancer. Markers of prognosis and tumor progression were considered as they affect management. Hormones and their cognate receptors, cytokines, as well as specific signal transduction pathways were all considered for the development of more specific breast cancer treatment. The consensus statement from the meeting follows.

\section{Prognostic and predictive markers}

There is general agreement that many women with primary breast cancer receive unnecessary treatment following surgery. For example, large meta analyses of randomized trials, and several series of uncontrolled, retrospective studies indicate that approximately $70 \%$ of women whose tumor has not spread to the axillary lymph nodes are alive and disease-free 10 years after surgery with no additional systemic chemotherapy or endocrine therapy. Unfortunately, the currently available prognostic factors, either alone or in combination, cannot accurately identify this large subset of patients who will derive very little benefit from additional adjuvant therapy. Given the proven clinical benefits of adjuvant therapy, even among subsets of patients with node-negative disease, it is increasingly difficult to conduct prospective clinical trials that include untreated control groups. Therefore, there is a need to retrospectively identify and collect tumor and non-malignant host tissue samples from large series of well-characterized patients who did not receive adjuvant therapy and for whom long-term follow-up is 
available. These types of tissues can be used to create molecular fingerprints of individual cancers as well as host factors using emerging molecular biology tools, which could help identify new panels of prognostic biomarkers. The combination of anatomic, biologic and molecular features could provide a more accurate assessment of the likely clinical course of an individual breast cancer, and help determine the most appropriate treatment strategy for an individual patient.

An area of concern that needs additional attention has arisen from our desire to improve the accuracy of existing breast cancer prognostic and predictive parameters. Retrospective studies of archived surgical specimens from previously staged and treated breast cancer patients now indicate that even the best of pathologists, using standard histologic techniques, are unable to discern millimeter-sized breast tumor nodal deposits in up to $20-30 \%$ of patients originally thought to have node-negative disease. This possible error in pathologic staging of node-negative breast cancers may account for the generally observed $\sim 20 \%$ metastatic relapse rate of patients with early-stage disease and needs to be further evaluated.

New therapies for breast cancer that target specific molecular pathways are currently being developed. An important use of biomarkers is the determination that the pathway is active and functional in a specific patient before therapy is given. A second use is to determine that the therapy has actually altered the pathway and has provided clinical benefit to the patient. This class of predictive biomarkers is growing rapidly, and new types of clinical trials will be required to truly validate their clinical utility. There is a significant need to standardize many assay methodologies for measuring biomarker levels. The development of immunoreagents suitable for use on paraffin-archived tissues and able to semi-quantitate ER/PR and HER2/ErbB2 receptor expression have clearly pointed to the need for more standardized and validated assay procedures to accurately assess any given biomarker. In particular, the commercial availability and community use of many different but unvalidated antibodies currently employed in PR and HER2/ErbB2 immunohistochemical assays have led to widespread discordances between the assay results and inaccurate biomarker scoring when assessed against identical test samples. Since predictive biomarkers are often used to identify patients eligible to receive selective biomarker-targeted therapeutics (e.g., endocrine therapy, or treatment with the
anti-HER2/ErbB2 antibody trastuzumab), inaccurate biomarker assays result in unacceptable personal and economic consequences. Assay development, standardization and validation should parallel the development and evaluation of new therapies, so those patients who will or will not respond to specific targeted therapies can be accurately identified. Appropriately, this particular issue is now receiving increased regulatory (e.g., FDA) attention.

Somewhat more distant on the translational research horizon are encouraging reports from several research groups who are employing thousands of newly available gene sequences from the Human Genome Project coupled with novel bioengineering advances that allow simultaneous quantification of the expression of all these genes from a given breast tumor on a single microscope slide-based assay. Such molecular profiling of breast cancers by gene expression microarrays appears to be confirming the existence of many hitherto unrecognized subsets of breast tumors; from these exciting results we expect to establish more accurate signatures of those breast tumors destined to metastasize and those most likely to respond to our best therapeutics.

\section{Steroid receptors}

Human mammary epithelial cells and many breast carcinomas express one or both of the two estrogen receptors, $\mathrm{ER} \alpha$ and $\mathrm{ER} \beta$. The single most important marker of estrogen sensitivity and response to tamoxifen therapy in breast cancer is the presence of $\mathrm{ER} \alpha$ in tumor cells. $\mathrm{ER} \alpha$ is also considered a significant target for breast cancer prevention by selective estrogen receptor modulators (SERMs) like tamoxifen and raloxifene, as demonstrated by the NSABP P1 and MORE trials. Both ER subtypes are presumably the targets of aromatase inhibitors, which have been shown to be at least as effective as tamoxifen for preventing or delaying recurrent hormone dependent disease. In addition, recent data suggest that the frequent occurrence of a somatic point mutation in $\mathrm{ER} \alpha(\mathrm{K} 303 \mathrm{R})$ that increases its sensitivity to estrogens is significantly associated both with breast hyperplasia and metastatic diseases. Thus, ER $\alpha$ remains a major target for breast cancer prevention and therapy and $\mathrm{ER} \beta$ is a potential target.

The expression, function and importance of ER $\beta$ in normal breast epithelium and in breast cancer are 
unclear at the present time. Estrogens and SERMs regulate diverse cellular activites via one or both of the two ER subtypes (ER $\alpha$ and $E R \beta)$ in hormone responsive tissues and cancers. Liganded ERs can interact with a complex mix of coactivators, corepressors and other signaling molecules that differ in expression and importance from tissue to tissue. In addition, different SERMs may alter the affinity and/or selectivity of one or both ERs for these coregulators, allowing for tissue-selective responses. There is also evidence that the two ER subtypes can have different actions in the same tissue and that they can interact with each other, which may result in complex responses to a given SERM. Recent ER $\alpha$ and $E R \beta$ structure information provides a partial molecular explanation for estrogen agonism and antagonism. The crystal structures of ER $\alpha$ and ER $\beta$ ligand binding domains (LBDs) complexed with a number of SERMs, ranging from full agonists (estradiol and diethylstilbestrol), to partial agonists (genistein), to mixed agonists/antagonists (raloxifene and tamoxifen), to complete antagonists (faslodex). This information has helped define and predict some of the properties and behaviors one might expect from different SERMs. However, our knowledge of the underlying mechanisms is far from complete. For example, dysregulation of the ER $\alpha$ coactivator AIB1 may result in over-stimulation of $\mathrm{ER} \alpha$ in some breast cancers. Also, it is likely that rapid, nongenomic actions of SERM-ER complexes are important and largely unstudied mechanisms by which SERMs regulate proliferation, apoptosis or other cellspecific responses to SERMs. Thus, the current challenges in this area are: (1) understanding the molecular mechanisms by which SERMs elicit tissue-selective agonist or antagonist responses via one or both ER subtypes, and (2) understanding the roles of $\mathrm{ER} \alpha$ and ER $\beta$ expression and modification in breast cancer genesis and progression to hormone independence.

It is thought that most invasive breast cancers develop over may years from premalignant lesions. Only a few of these benign lesions, such as usual ductal hyperplasia or atypical hyperplasia, appear to have significant premalignant potential. Unfortunately, our understanding of the underlying biology and clinical course of these lesions is incomplete. Based on epidemiological evidence, breast cancer evolution has been proposed in which normal ductal epithelium evolves to usual and atypical hyperplasia, to carcinoma in situ, to invasive, and finally to metastatic cancer. Hyperplasias are considered weak risk factors for developing invasive breast cancer (approximately
2-5 fold), with increasing risks seen for carcinoma in situ (10-fold). It has also been proposed that many of these premalignant lesions never progress to invasive cancer, so their progression can be viewed as nonobligatory, presumably requiring the accumulation of additional genetic events to facilitate eventual tumor development.

How might these additional genetic events accumulate? One key factor thought to play a role in both the development and progression of these early lesions is estrogen. Estrogens are important for regulating the proliferation of breast cancer and premalignant lesions. Estrogens affect cellular processes by binding to their cognate receptors, $\mathrm{ER} \alpha$ and $\mathrm{ER} \beta$, and function as transcription factors to mediate the mitogenic effects of estrogen. One hallmark of premalignant lesions is an increase in ER $\alpha$ expression. The relative overexpression of $\mathrm{ER} \alpha$ early in breast cancer evolution could conceivably influence its course via inappropriate proliferation, and potential accumulation of genetic alterations. Thus, we hypothesize that inappropriate overexpression of wild-type $\mathrm{ER} \alpha$ or the emergence of a mutated $\mathrm{ER} \alpha$ driving proliferation could be early molecular events in the evolution of premalignant breast disease. Furthermore, since the ER status of breast cancer metastases usually correlates with the ER status of the primary tumor, an emerging area of research is focused on understanding the role that ER may play in the metastatic progression of breast cancers. It is conceivable that early in breast cancer progression, ER-negativity could be considered a virulence factor, but later in the disease dissemination, ER-positive tumors, especially those with a mutant form of the $\mathrm{ER} \alpha(\mathrm{K} 303 \mathrm{R})$, may play another role in the metastagenicity of breast cancer. Studies examining these 'new' roles for ER in breast cancer are warranted.

Therefore additional molecular, structural and functional information is needed to improve our knowledge of how $\mathrm{ER} \alpha$ and $\mathrm{ER} \beta$ mediate individual and collective responses to SERMs, especially in concert with intersecting growth factor pathways and with coactivators and corepressors that may be inappropriately up- or down-regulated. More information is also needed about the expression and roles of $\mathrm{ER} \alpha, \mathrm{ER} \beta$ and any mutant forms of these receptors in normal breast epithelium and in progressive breast disease. The development of novel SERMs that can elicit ER subtype-selective responses, both agonisitic and antagonistic, should also be an important goal for therapeutic or preventive intervention. 


\section{Potential clinical application of glucocorticoid receptor activation in breast cancer}

The role of nuclear hormone receptors in the development of breast cancers has been the subject of intense interest. The majority of studies have focused on the function and expression of estrogen, progesterone and to a lesser extent, prolactin receptors in mammary gland development and function. Despite the ubiquitous nature of glucocorticoid receptor (GR) expression in normal and malignant mammary epithelium, the GR has received relatively little consideration as a potential signaling pathway in breast cancer. However, recent data from transgenic mice and cell culture experiments using human malignant and non-malignant breast epithelium point to an important role for the GR in both mammary gland development and survival signaling of breast epithelial cells.

Using transgenic mice models that substitute a mutant GR receptor for the wild type receptor, it has been shown that a lack of GR DNA binding function impairs the ductal development of the mammary gland in virgin females, although the mechanism (e.g., a proliferative versus apoptotic defect) is not established. The GR is also known to be required for normal lactation in adult mice, however, the GR's DNA binding function does not appear to be required for this function. Recently, investigators from several laboratories have demonstrated a potent in vitro survival effect from GR activation in mammary epithelial cells subjected to growth factor deprivation. The role of glucocorticoid in inhibiting paclitaxelmediated cell death in breast cancer cell lines has also been recently demonstrated. Together, these observations raise the interesting possibility that GR activation may have an important anti-apoptotic role in mammary epithelial cell biology. In view of the pharmacologic doses of glucocorticoid that are routinely administered prior to chemotherapy in breast cancer patients, as well as the potentially high local levels of endogenous corticosteroids produced by adipose tissue in the human breast, the role of GR activation in breast cancer signaling should be studied further. Such studies should encompass both a basic laboratory approach as well as attention to the potential effects that glucocorticoid therapy may have on the effectiveness of cytotoxic treatment in breast cancer. If GR activation is confirmed to play an important role in breast epithelial survival signaling GR, antagonists may eventually be explored as a novel therapeutic modality for breast cancer.

\section{Targeted therapy of breast cancer}

It is increasingly apparent that the term 'breast cancer' describes a population of diseases with elements of functional commonality and interrelated but distinct molecular genotypes and phenotypes. Patterns of hormone and growth factor receptor polymorphisms, expression, overexpression and mutation define some of these phenotypes, and help to define new therapeutic targets. In recent years, much interest has focused on the epidermal growth factor receptor family, with particular emphasis on HER2/Erb2/neu as a therapeutic target. It is likely that important new treatment options will emerge as new cell surfaceassociated receptor targets are identified and validated. However, multiple lines of evidence now indicate that the net effect of perturbations in growth factor receptor-mediated signaling is to inhibit the activation of key intracellular kinases such as AKT. These findings suggest two potentially complementary approaches to the therapeutic inhibition of breast cancer cell signaling. In the first instance, the function of the downstream kinase can be directly inhibited; this will have important therapeutic implications if this can be accomplished with functional selectivity for malignant as opposed to normal cells. In the second scenario, the direct inhibition of the targeted kinase will be unacceptably toxic to normal cells. If so, the only way to achieve therapeutic inhibition of that kinase will be to selectively inhibit the cancer-related signaling elements that influence the phosphorylation of that kinase. Defined combinations of upstream or associated pathway inhibitors tailored to the molecular phenotype of a particular cancer can be employed to create an indirect or 'summation inhibition effect' of the targeted downstream kinase. Thus, the targeted kinase is selectively inhibited in cancer cells, but in fewer normal cell types. Irrespective of the means by which targeted therapy exerts its anti-tumor effects, the successful implementation of this evolving approach is likely to include combination with classic cytotoxic chemotherapy agents.

\section{The development of herbals and other natural products as anti-cancer agent}

There is a need for agents with greater efficacy and decreased toxicity for the treatment and prevention of breast cancer. The majority of agents developed to date are either natural products, such as vincas and taxanes, 
antimetabolites or alkylators. Most of these agents have either been found by empiric screening of natural products or synthesized chemicals. Recently, with the identification of potentially more specific molecular targets for therapy, a few agents have been developed rationally, including trastuzumab and several novel SERMs. It seems clear that with the identification and validation of more targets for therapy, the need for agents that specifically block or inhibit the targets or pathways of interest will become even more important.

Many patients seeking additional gains have used a wide variety of alternative therapies including various nutritional supplements and herbal remedies. In some cultures, the latter are considered a legitimate alternative to more traditional Western pharmaceuticals. Unfortunately, the development of these herbal remedies as validated means of treating cancer has been hampered for several reasons. First, most of hese herbal remedies exist as inexact formulations of multiple components, each of which may, in and of itself, be an impure extract. Second, few, if any, such herbals have been subjected to rigorous evaluation using credible trial designs. Third, the mechanisms of action for herbal agents are rarely known. Possibly, the greater the difficulty in establishing an intellectual property position for some of these agents may have also slowed their development by pharmaceutical companies. Nonetheless, there is widespread belief amongst patients, as well as healthcare professionals, that significant activities are to be found buried in these complicated mixtures of agents.

We believe that this area of investigation needs substantially more attention. The individual chemical moieties contained within these mixtures need to be identified and structurally analyzed. Modern pharmacophore methodologies suggest that uniquely defined ingredients contained within these herbal remedies can be 'matched' to specific targets whose structures have been solved by crystallography or NMR means. It is also critical that defined chemicals within such herbal teas be used in traditional empiric screens employed by the NCI as well as pharmaceutical companies. Finally, it is essential that agents which show in vitro activity be evaluated either in formal, well-designed clinical trials of clinical efficacy or explored by approaches using surrogate endpoints of response, so that evidence of efficacy can be obtained efficiently.

\section{Progression and prognosis in breast cancer}

Cancer is a disease involving dynamic changes in the genome. Multiple steps occur in the progression from a normal cell to the malignant phenotype, and further progression and changes occur even in an already malignant cell and the clinically apparent tumor. These changes account for the heterogeneity in the clinical presentation of cancer, and the differences in outcome in seemingly uniform patient populations. The understanding of the molecular characteristics of this progression may allow a better individualizing of therapy.

Multiple genotyping changes are needed to develop traits that are necessary for cancer progression. Epigenetic mechanisms, as well as extracellular signals, contribute to tumor phenotype. Tumors may undergo different permutations of genetic events to arrive at the necessary phenotype. Genotype identifies the malignant traits but the mutational paths to a specific phenotype are varied and redundant. Convergent evolution requires that only certain essential phenotypes be acquired, hence evolutionary selection determines the dominant mutational pathways and resulting phenotype.

Expression of molecular markers of metastatic progression can be determined in human breast cancer specimens obtained at surgery, and they can be correlated with the known clinical outcome. The important phenotypes necessary for cancer progression are growth, neovascularization, decreased homophilic adhesion, motility, invasion and metastasis. All these traits can be determined on tissue. Early markers are likely those that are expressed in the majority of tumors and these are not contributory to prognosis because they would be present in all cases. Angiogenesis and expression or urokinase plasminogen activator, for example, are high in most node-negative breast cancers and therefore have little overall prognostic value. In contrast, loss of nm23, abnormal p53, increase in MMP-2 and decrease in E-cadherin which occur in progressively smaller proportions of node-negative breast cancers at diagnosis and represent phenotypes of more advanced disease progression are therefore expected to have greater prognostic impact.

Address for offprints and correspondence: Samuel Hellman, The University of Chicago, 5758 So. Maryland Ave., MC 9006, Chicago, IL 60637, USA; Tel.: +1-773-702-4346; Fax: +1-773702-4347; E-mail: s-hellman@uchicago.edu 